\section{Horizontal Gene Transfer}

\section{Rajarapu G*}

Department of life sciences, University of Wolverhampton, United Kingdom

\section{Horizontal Gene Transfer in Eukaryotes}

Horizontal gene transfer (HGT) which is also called as lateral gene transfer is a non-sexual moment of genetic information between the two organisms. Across the eukaryotes the impact of HGT was irregular. Some of the plant mitochondria and many microbial eukaryotes are good examples for HGT in eukaryotes. For acquiring the new genes some other lineages are appearing to be immune. Another important pattern in eukaryotes is in HGT eukaryotes involves the genes from the bacteria. This is because of two reasons one is because of sampling and other is due to ecological factors. There are several ways to detect HGT but the standard way to find is phylogenetic analysis because our expectation does not match to this [1].

\section{Types of HGT in Eukaryotes}

\section{Simple transfer}

It shows the simple moving of the genes from bacteria to eukaryotes and replaces prevailing homologue. In phylogenetic tree it shows that eukaryotic gene is far away from the other eukaryotes and it was closely related to bacterial lineage. It is easy method of HGT Figure 1.

\section{Serial transfer}

It shows the complex serial transfer of genes. Red colour indicates the bacterial gene which moved into eukaryotes (Blue colour). From the first eukaryote recipient second eukaryote has acquired a gene which results in two closely related eukaryotes are sharing the single gene. Irregular transfer shows that genes are from various organism and they are not closely related Figure 2 [1].

\section{Novel gene transfer}

Irregular transfer of genes is observed in this type. Finding new function is an important aspect in this type of transfer. Irregular transfer shows that genes are from various organism and they are not closely related Figure 3 [1].

\section{Importance of HGT}

It is considered as vital strength in evolution of bacteria. Many of the eukaryotic are sequenced. Antibiotic resistance is the main feature of HGT [2].
Organism tree

(a) Simple transfer

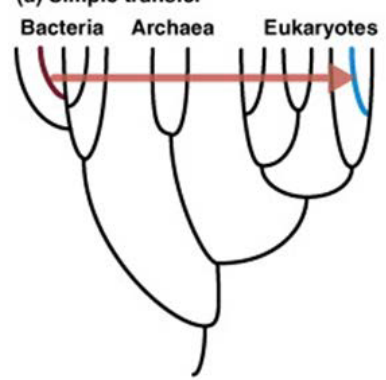

Gene tree

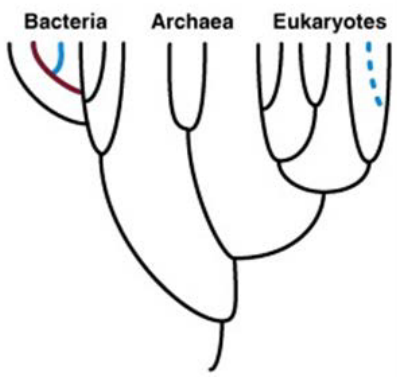

Figure 1: Simple horizontal gene transfer (Keeling, 2009).
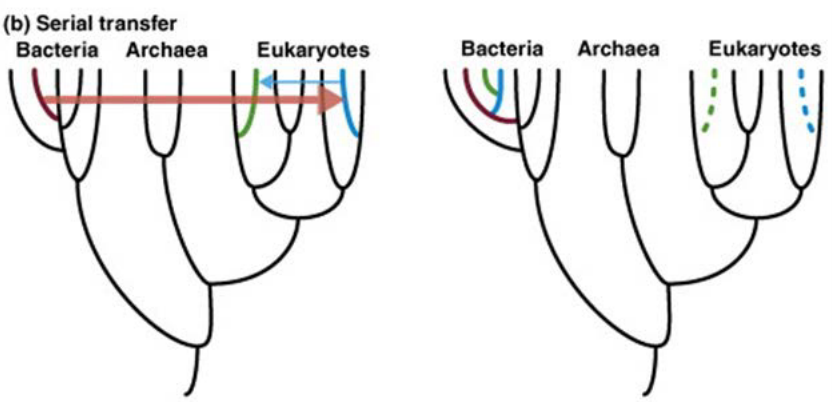

Figure 2: Serial horizontal gene transfer (Keeling, 2009).
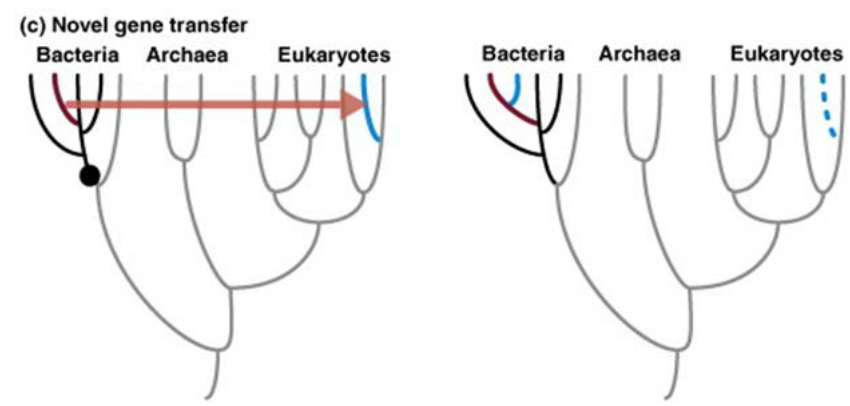

Figure 3: Novel horizontal gene transfer (Keeling, 2009).

\section{Disease emergence between eukaryotes through HGT}

Horizontal gene transfer is poorly understood in different species of eukaryotes. But from the studies of Friensen and his colleagues states that between the two species of fungi HGT allowed the species to acquire the high virulence on wheat. In prokaryotes HGT is well known. There are some evidences which convincing that HGT occurs between the bacteria to eukaryotes and within eukaryotes to eukaryotes. In many multicellular eukaryotes the separation of germ and soma is believed to limit horizontal gene transfer. Separation of germ and soma does not exist in many eukaryotic microorganisms. Many horizontal gene transfer cases are suspected in fungi. But it is difficult assessed their impact on fungal ecology and evolution this is because this gene transfers are taken place ling back ago [3].

\section{Detecting and evaluating cases of HGT}

Several methods or approaches are there to suspect that HGT was

*Corresponding author: Rajarapu G, Department of life sciences, University of Wolverhampton, United Kingdom, Tel: 07404307002; E-mail: rajarapu.wlv@gmail.com

Received September 22, 2014; Accepted October 17, 2014; Published October 20, 2014

Citation: Rajarapu G (2014) Horizontal Gene Transfer. J Phylogen Evolution Biol 2: 136. doi:10.4172/2329-9002.1000136

Copyright: () 2014 Rajarapu G. This is an open-access article distributed under the terms of the Creative Commons Attribution License, which permits unrestricted use, distribution, and reproduction in any medium, provided the original author and source are credited. 
occurred. But to identify the potential cases of HGT these methods were not sufficient unless it was followed up by phylogenetic analysis. In the year 2001 one of the published claim is draft sequence of human genome was containing 200 genes that are identical to bacteria. To clarify this claim with the human gene top blast hits were used. To this draft sequences the closed one were bacterial homologues. Interesting step in this pattern is this relationship was neglected by the phylogenetic analysis. Horizontal gene transfer is also involved in some cases in which human genes have homologues in bacteria but not in eukaryote genome which are completely sequenced. For identifying the HGT the gold and standard method was phylogenetic analysis. In horizontal gene transfer phylogenetic is the important method because it will occur when there is a strong conflict between the organism and phylogeny of the gene [4].

\section{Horizontal Gene Transfer in Eukaryotic Evolution}

\section{Prokaryote to eukaryote transfer}

Genes that are acquired from the bacterial sources are varied in different species. The number may be zero to hundred. For the evidence of bacterial horizontal gene transfer few genomes are equally analyzed from organisms. Six genomes are sequenced from the land plants and several genomes from animals are sequenced for analysis. In all this cases reliable cases of bacterial horizontal gene transfer was not found. Some of the fungal genomes are also sequenced symmetrically in which analysis results show that they have some bacterial genes. In most of the genomes that are studied the important limitation is search strategies with initial steps. Studies are started with BLAST search in which from the given genome genes have to identify the gens that are having similar blast hits with bacterial genes and it is followed by phylogenetic analysis (or) identifying the genes from the organism which is under study relative to eukaryotes or its related organism [4].

\section{Eukaryotes to prokaryotes transfer}

Horizontal gene transfer from some eukaryotes cases has been reported. Interesting factor is transferred genes are identified in eukaryote organisms. In the gene transfer proteins play major role in this which imparts some important functions in hosts. For example in the bacteria Prosthecobacter alpha and beta tubulins are encode by operon which includes other eukaryotic gene [4].

\section{Polyphosphate Kinases (PPK)}

Polyphosphate kinase is a polymer of hundreds of phosphate residues. In many bacteria main polyphosphate kinase gene is ppk gene which is detected in eukaryotes also. In eukaryotes poly phosphate kinase is less widely studied. Polyphosphate kinase enzyme is main poly synthetic in many organisms. Polyphosphate kinase encoding genes are ppk-1 and ppk-2 in this PPK-1 the synthetic enzyme and PPK-2 is involved in GTP production [5].

Polyphosphate kinase is ubiquitous in nature. In many of the organisms (algae, fungi, plants, insects, and animals) PPK is a dynamic molecule. Polyphosphate kinase plays an important role in organism's metabolic functions. Some of the important functions of polyphosphate kinases are motility, biofilm formation, blood coagulation, cancer cell proliferation etc. for example in E.coli polyphosphate kinase is one of the parts in poly hydroxybutryate $\mathrm{Ca} 2+$ complex which is responsible for uptake of DNA. PPK are identified in many prokaryotes and few in eukaryotes [6].

More than hundred bacterial species in several pathogens were recognised during past decade which are having PPK1 and PPK2.
Structure of PPK1 contains ATP binding site at the site where ATP is normally binding contains analogue AMMPPNP which contains the growing poly $\mathrm{P}$ chain. For investigators the structural information of PPK1 will help in developing the compounds which blocks the binding sites. In animal cells absence of PPK1 active site makes the toxicity of inhibitory drugs. PPK2 is also found in several bacterial species and pathogens. In these some bacterial species are with both PPK1 and PPK2 sequences. Mostly PPK2 sequence is found in archea, rhizobia, cyanobacteria, and Streptomyces spp. [7].

\section{Phylogenetic Analysis}

For the many research areas in biology such as comparative genomics, functional prediction, detection of lateral gene etc. phylogenetic analysis is the central and main part. Phylogenetic analysis mainly concerned with the identification of homologous sequence, their multiple alignment, phylogenetic reconstruction and graphical representation of infrared tree. Phylogeny.fr is a new program which is developed for non-specialist in phylogenetic analysis. Phylogeny. fr automatically performs all the tasks such as homologous sequence, their multiple alignment, phylogenetic reconstruction and graphical representation of infrared tree. As phylogeny.fr was designed for nonspecialists but it also meet the needs of specialists non-specialists in phylogenetic analysis will look for up to date tools in phylogeny to analyses their data in healthy way, while the specialists will run sophisticated analysis easily. For analysis of data phylogeny.fr provides three types of modes. For targeting the non-specialists 'One-click' mode was developed it provides up to date phylogeny pipeline (from a set of sequences it allows to build phylogenetic tree at once.) chain program with speed and accuracy. Some of the examples for one-click mode are as fallows

- $\quad$ MUSCLE for multiple alignments.

- $\quad$ phyML for tree building.

- TreeDyn for tree rendering.

This program is set with all the parameter the user has to do is input the sequence to get a tree. Second mode is 'Advanced mode' it also have same program as the first one but the user has to modify the parameters of the each program. Third mode is 'A la carte' which is more flexible and sophisticated mode. In this mode to meet the specification user can setup own pipeline by selecting and setting from a general or specialised data and available tools in large choice. By running the blast user can also collect the data [8].

\section{Tree View}

Tree view is an application through which phylogenetic tree is displayed on a personal computer. It runs on both MacOS and windows the two common personal computers used by many biologists. Some other tree view programs (Mac clade, PAUP) will provide good facilities but they are unable to run in Apple Macintosh computers. Draw gram and draw tree are other programs for tree view which will run in both MacOS and windows but they are not good enough to utilize the graphical representation under the operating systems. In both windows and MacOS tree view program runs and allows the users to use the formats installed in their machines and printers. For pasting picture on other applications (or) for creating graphic files tree view program support the graphic file format. Regular file operations such as drag and drop are also supported by tree view. Tree view program can able to read a range of tree file formats and in different ways of style formats it can displays the tree. Beside of this tree view can also display some additional information like internal node labels, edge lengths etc. if the 
tree file format is present in more than one tree with the help of tree view user can view each tree separately and able to save the format in a different file formats [8].

Research works on phylogenetic analysis reveals that many of the eukaryotes genes whose ancestry is prokaryotes are from bacterial origin. Most of these eukaryotic genes and their products show functions in cytosolic metabolism. Results of phylogenetic analysis are surprising that eukaryotic nuclear genome and archaeal genome shares most common ancestor. Some of the bacterial genes were expected during the transfer from mitochondria, but when these genes are reimported in to mitochondria they show limited functions. One thing we should notice here is presence of bacteria genes with various functions should not be a surprise. Results of gene transfer notice that eukaryotic genes were replaced by genes of bacteria which are taken as food by them [9].

\section{Phylogeny.fr}

For the many research areas in biology such as comparative genomics, functional prediction, detection of lateral gene etc. phylogenetic analysis is the central and main part. Phylogenetic analysis mainly concerned with the identification of homologous sequence, their multiple alignment, phylogenetic reconstruction and graphical representation of infrared tree. Phylogeny.fr is a new program which is developed for non-specialist in phylogenetic analysis. Phylogeny. fr automatically performs all the tasks such as homologous sequence, their multiple alignment, phylogenetic reconstruction and graphical representation of infrared tree. As phylogeny.fr was designed for nonspecialists but it also meet the needs of specialists non-specialists in phylogenetic analysis will look for up to date tools in phylogeny to analyse their data in healthy way, while the specialists will run sophisticated analysis easily. For analysis of data phylogeny.fr provides three types of modes. For targeting the non-specialists 'One-click' mode was developed it provides up to date phylogeny pipeline (from a set of sequences it allows to build phylogenetic tree at once.) chain program with speed and accuracy. Some of the examples for one-click mode are as fallows

- $\quad$ MUSCLE for multiple alignments.

- $\quad$ phyML for tree building.

- TreeDyn for tree rendering.

This program is set with all the parameter the user has to do is input the sequence to get a tree. Second mode is 'Advanced mode' it also have same program as the first one but the user has to modify the parameters of the each program. Third mode is 'A la carte' which is more flexible and sophisticated mode. In this mode to meet the specification user can setup own pipeline by selecting and setting from a general or specialised data and available tools in large choice. By running the blast user can also collect the data [7].

\section{Discussion}

In eukaryotes for evidence of horizontal gene transfer of bacteria type PPK1 and PPK2 180-200 eukaryotic genomes have been examined. In any particular database distinct list of bacterial type PPK is not seen. Research works shows presence of few eukaryotic PPK1 enzymes but no matches of PPK2 enzymes was found. According to Hooley, et al., 2008 small number of bacteria type PPK1 and PPK2 is present in eukaryotes. PPK genes of eukaryotes which are lacking in introns show similarities with prokaryotic organisms in general prokaryotes do not contain introns. $\mathrm{N}$-terminal domain is absent in T.trahens because of absence of DNA sequences encoding which was seen in earlier eukaryotes.
Misleading gene description is taken place because of errors in DNA sequences and annotation [9].

For horizontally transferred PPK1's in photosynthetic organisms cyanobacteria are the donor bacteria. Photosynthetic organisms are those which generate chloroplasts by endosymbiosis examples are P.patens and the green algae O.lucimarinus and O.tauri. In P.pallidum, T.trahens and Dictyostelium species phygocytosed bacteria is donor species for gene encoding PPK enzymes. For N.vectensis which is also called as sea anemone the donor species are phygocytosed bacteria.

Among the sequenced eukaryotes O.tauri and O.lucimarinus are most divergent species in same genus these are because of $70 \%$ of identity in amino acid composition. Though horizontal gene transfer was occurred in algal evolution when we predicted the transferred gene it was not looking as horizontally transferred gene. Because of this it is difficult to trace a common or single origin of specific PPK gene [9].

In eukaryotic algal evolution the ole of HGT was proposed by Raymond and Blankenship (2003). At different times aciquation of new genes in the species by engulfment (endosymbiosis) of cyanobacteria (Raymond and Blankenship, 2003). In the marine environment other examples of horizontal gene transfer in to metazones are between plants and animals. By the absence of Eukaryotic PPKs certain organisms are highlighted. Metabolism is affected in several fungal species because of horizontal gene transfer.

Spliceosomal introns functions are not known, but their presence in nuclear genome shows great role in evolution (Roy and Irimia, 2009). Short life cycle of organisms is proportionate to less intronisation and intron length to gene expression in unicellular and multicellular organism is positive and negatively correlated respectively [9].

\section{References}

1. Patrick JK (2009) Functional and ecological impacts of horizontal gene transfer in eukaryotes. Current Opinion in Genetics \& Development 19: 613-619.

2. John WW, Glenn AM, David RW (2009) Prediction of horizontal gene transfers in eukaryotes: approaches and challenges. Biochemical Society Transactions 37: 792-795

3. Ian RS (2006) Rapid disease emergence through horizontal gene transfer between eukaryotes. Trends in Ecology and Evolution 21: 656-658.

4. Patrick JK, Jeffrey DP (2008) Horizontal gene transfer in eukaryotic evolution Nature reviews genetics 9: 605-618

5. Paul Hooley, Michael PW, Michael RWB (2008) Eukaryote polyphosphate kinases: is the 'Kornberg' complex ubiquitous?. Trends in Biochemical Sciences 33: $577-582$

6. Haiyu Zhang, Marı'a R, Xiaobing Shi, Narayana NR, Arthur Kornberg (2007) Polyphosphate kinase 1, a conserved bacterial. PNAS 104: 16486-16491

7. Tree View.

8. Dereeper A, Guignon V, Blanc G, Audic S, Buffet S, et al. (2008) Phylogeny fr: robust phylogenetic analysis for the non-specialist. Nucleic Acids Research 36: W465-W69

9. Doolittle WF (1998) You are what you eat a gene transfer ratchet could account for bacterial genes in eukaryotic nuclear genomes. Trends in Genetics 14: 307311. 